\title{
Combining Column Generation and Lagrangian Relaxation
}

\author{
Dennis Huisman, Raf Jans, Marc Peeters and Albert P.M. Wagelmans
}

\begin{tabular}{|l|l|}
\hline \multicolumn{2}{|l|}{ ERIM REPORT SERIES RESEARCH IN MANAGEMENT } \\
\hline ERIM Report Series reference number & ERS-2003-092-LIS \\
\hline Publication & 2003 \\
\hline Number of pages & 23 \\
\hline Email address corresponding author & huisman@few.eur.nl \\
\hline Address & Erasmus Research Institute of Management (ERIM) \\
& Rotterdam School of Management / Rotterdam School of \\
& Economics \\
& Erasmus Universiteit Rotterdam \\
& P.O. Box 1738 \\
& 3000 DR Rotterdam, The Netherlands \\
& Phone: +31 10 408 1182 \\
& Fax: $\quad+31104089640$ \\
& Email: info@erim.eur.nl \\
& Internet: www.erim.eur.nl \\
\hline
\end{tabular}

Bibliographic data and classifications of all the ERIM reports are also available on the ERIM website: www.erim.eur.nl 


\title{
ERASMUS RESEARCH INSTITUTE OF MANAGEMENT
}

\author{
REPORT SERIES \\ RESEARCH IN MANAGEMENT
}

\begin{tabular}{|c|c|c|}
\hline \multicolumn{3}{|c|}{ BIBLIOGRAPHIC DATA AND CLASSIFICATIONS } \\
\hline Abstract & \multicolumn{2}{|c|}{$\begin{array}{l}\text { Although the possibility to combine column generation and Lagrangian relaxation has been } \\
\text { known for quite some time, it has only recently been exploited in algorithms. In this paper, we } \\
\text { discuss ways of combining these techniques. We focus on solving the LP relaxation of the } \\
\text { Dantzig-Wolfe master problem. In a first approach we apply Lagrangian relaxation directly to this } \\
\text { extended formulation, i.e. no simplex method is used. In a second one, we use Lagrangian } \\
\text { relaxation to generate new columns, that is Lagrangian relaxation is applied to the compact for- } \\
\text { mulation. We will illustrate the ideas behind these algorithms with an application in Lot-sizing. To } \\
\text { show the wide applicability of these techniques, we also discuss applications in integrated } \\
\text { vehicle and crew scheduling, plant location and cutting stock problems. }\end{array}$} \\
\hline \multirow{3}{*}{$\begin{array}{l}\text { Library of Congress } \\
\text { Classification } \\
\text { (LCC) }\end{array}$} & $5001-6182$ & Business \\
\hline & $5201-5982$ & Business Science \\
\hline & HB 143.7 & Optimization Techniquese \\
\hline \multirow{3}{*}{$\begin{array}{l}\text { Journal of Economic } \\
\text { Literature } \\
\text { (JEL) }\end{array}$} & M & Business Administration and Business Economics \\
\hline & $\begin{array}{l}\text { M } 11 \\
\text { R } 4\end{array}$ & $\begin{array}{l}\text { Production Management } \\
\text { Transportation Systems }\end{array}$ \\
\hline & C 61 & Optimization Techniques \\
\hline \multirow{3}{*}{$\begin{array}{l}\text { European Business Schools } \\
\text { Library Group } \\
\text { (EBSLG) }\end{array}$} & $85 \mathrm{~A}$ & Business General \\
\hline & $\begin{array}{l}260 \mathrm{~K} \\
240 \mathrm{~B}\end{array}$ & $\begin{array}{l}\text { Logistics } \\
\text { Information Systems Management }\end{array}$ \\
\hline & $255 \mathrm{~A}$ & Decision theory \\
\hline \multicolumn{3}{|c|}{ Gemeenschappelijke Onderwerpsontsluiting (GOO) } \\
\hline \multirow[t]{3}{*}{ Classification GOO } & 85.00 & Bedrijfskunde, Organisatiekunde: algemeen \\
\hline & $\begin{array}{l}85.34 \\
85.20\end{array}$ & $\begin{array}{l}\text { Logistiek management } \\
\text { Bestuurlijke informatie, informatieverzorging }\end{array}$ \\
\hline & 31.80 & Toepassingen van de wiskunde \\
\hline \multirow[t]{3}{*}{ Keywords GOO } & \multicolumn{2}{|c|}{ Bedrijfskunde / Bedrijfseconomie } \\
\hline & \multicolumn{2}{|c|}{ Bedrijfsprocessen, logistiek, management informatiesystemen } \\
\hline & \multicolumn{2}{|c|}{ Lagrange functies, series, roosters, bemanningen, rijtuigen, voorraadbeheer } \\
\hline Free keywords & \multicolumn{2}{|c|}{$\begin{array}{l}\text { Column generation, Lagrangean relaxation, lotsizing, vehicle and crew scheduling, cutting stock } \\
\text { problem }\end{array}$} \\
\hline
\end{tabular}




\title{
Chapter 1
}

\section{COMBINING COLUMN GENERATION AND LAGRANGIAN RELAXATION}

\author{
Dennis Huisman \\ Erasmus Center for Optimization in Public Transport (ECOPT) and \\ Econometric Institute, Erasmus University Rotterdam, \\ P.O. Box 1738, NL-3000 DR Rotterdam, The Netherlands \\ Corresponding author \\ huisman@few.eur.nl
}

\section{Raf Jans}

Rotterdam School of Management, Erasmus University Rotterdam, P.O. Box 1738, NL-3000 DR Rotterdam, The Netherlands

rjans@fbk.eur.nl

Marc Peeters

Electrabel,

Place de L'Université 16, 1348 Louvain-La-Neuve, Belgium

Marc.Peeters2@electrabel.com

Albert P.M. Wagelmans

Erasmus Center for Optimization in Public Transport (ECOPT) and Econometric Institute, Erasmus University Rotterdam, P.O. Box 1738, NL-3000 DR Rotterdam, The Netherlands

wagelmans@few.eur.nl

\begin{abstract}
Although the possibility to combine column generation and Lagrangian relaxation has been known for quite some time, it has only recently been exploited in algorithms. In this paper, we discuss ways of combining these techniques. We focus on solving the LP relaxation of the Dantzig-Wolfe master problem. In a first approach we apply Lagrangian relaxation directly to this extended formulation, i.e. no simplex method
\end{abstract}




\begin{abstract}
is used. In a second one, we use Lagrangian relaxation to generate new columns, that is Lagrangian relaxation is applied to the compact formulation. We will illustrate the ideas behind these algorithms with an application in Lot-sizing. To show the wide applicability of these techniques, we also discuss applications in integrated vehicle and crew scheduling, plant location and cutting stock problems.
\end{abstract}

\title{
1. Introduction
}

In this chapter we consider (Mixed) Integer Programming problems in minimization form. Obviously, lower bounds for such problems can be computed through straightforward LP relaxation. Dantzig-Wolfe decomposition and Lagrangian relaxation are alternative methods for obtaining tighter lower bounds. The key idea of Dantzig-Wolfe decomposition (Dantzig and Wolfe (1960)) is to reformulate the problem by substituting the original variables with a convex combination of the extreme points of a substructure of the formulation. This results in the master or extended formulation, which contains the linking constraints from the original compact formulation and additional convexity constraints. When solving the LP relaxation of the master problem, column generation is used to deal with the large number of variables. Starting with a restricted master which contains only a small subset of all columns, we generate the other columns when they are needed. This is done by solving a so called pricing problem in which one or more variables with negative reduced costs are determined. After each execution of the pricing procedure, we calculate the optimal value of the LP relaxation of the restricted master, $\bar{v}_{R D W}$. This provides an upper bound on the optimal value of the Dantzig-Wolfe relaxation, $\bar{v}_{D W}$, which itself is a lower bound for the optimal IP value $v_{P}$. When a simplex algorithm is used to solve the restricted master, we obtain optimal values of the dual variables corresponding to the linking and convexity constraints. These values are used in the pricing problem to check if we can generate new columns with negative reduced cost. If we find such columns, we add them to the relaxed master and reoptimize, otherwise we have found the optimal Dantzig-Wolfe relaxation $\bar{v}_{D W}$. This value will usually be tighter than $\bar{v}_{P}$, the value of the LP relaxation of the original compact formulation.

In Lagrangian relaxation, the complicating constraints are dualized into the objective function. Given a specific vector of positive multipliers $u$, the Lagrangian relaxation problem always gives a lower bound, $\bar{v}_{L R}(u)$, on the optimal IP value $v_{P}$. The Lagrangian Dual problem consists of finding the maximum lower bound: $\bar{v}_{L D}=\max _{u \geq 0} \bar{v}_{L R}(u)$. Typically, the latter problem is solved using an iterative procedure, where in subsequent iterations, the Lagrangian multiplier vector $u$ is updated and 
we solve a new Lagrangian problem with these updated multipliers. In this chapter we focus on the subgradient method (e.g. Fisher (1985)) for approximating the optimal multipliers, although more advanced methods such as the bundle method (e.g. Lemaréchal, Nemirovskii and Nesterov (1995)) or the volume algorithm (Barahona and Anbil (2000)) exist.

There exists a strong relationship between Dantzig-Wolfe decomposition and Lagrangian relaxation. It is well known that when the Lagrangian relaxation is obtained by dualizing exactly those constraints that are the linking constraints in the Dantzig-Wolfe reformulation, the optimal values of the Lagrangian Dual, $\bar{v}_{L D}$, and the LP relaxation of the Dantzig-Wolfe reformulation, $\bar{v}_{D W}$, are the same. In fact, one formulation is the dual of the other (Geoffrion (1974), Fisher (1981)). Furthermore, the optimal dual variables $\mu$ for the linking constraints in the master correspond to optimal multipliers $u$ for the dualized constraints in the Lagrangian relaxation (Magnanti, Shapiro and Wagner (1976)). Moreover, the subproblem that we need to solve in the column generation procedure is the same as the one we have to solve for the Lagrangian relaxation except for a constant in the objective function. In the column generation procedure, the values for the dual variables are obtained by solving the LP relaxation of the restricted master, whereas in the Lagrangian relaxation, the Lagrangian multipliers are updated by subgradient optimization.

Both approaches have advantages and disadvantages. Lagrangian relaxation provides a lower bound on the optimal IP value $v_{P}$, but no primal solution is available. In addition, there are problems with the convergence of the subgradient algorithm. Usually the procedure is stopped after a fixed number of iterations, without the guarantee of having found the optimal value $\bar{v}_{L D}$ (Fisher (1985)). However, the subgradient optimization for updating the Lagrangian multipliers is computationally inexpensive and easy to implement. Column generation for solving the Dantzig-Wolfe reformulation, on the other hand, provides a primal solution at each iteration, which can be used to construct feasible solutions for the (M)IP in a rounding heuristic. However, simplex optimization of the master is computationally expensive and a tailing-off effect, i.e. slow convergence towards the optimum in the final phase of the algorithm, is generally observed (Barnhart et al. (1998), Vanderbeck and Wolsey (1996)).

In this chapter we will discuss how the relationship between DantzigWolfe decomposition and Lagrangian relaxation can be exploited to develop improved algorithms combining the strengths of both methods. We discuss two ways in which the two techniques can be combined effi- 
ciently. To be more specific, Lagrangian relaxation can be applied to the master problem to approximate optimal values of the dual variables or it can be used on the original compact formulation of the problem to generate good columns. However, notice that we will only discuss column generation within the framework of DW decomposition, but it can also be considered as a general LP pricing technique. For the combination of column generation and Lagrangian relaxation within this framework, we refer to Löbel (1998) and Fischetti and Toth (1997). In order to explain the general principles within the framework of DW decomposition in Section 1.2, we use the example of Capacitated Lot-sizing. In Sections 1.3-1.5, other applications and their specific implementation issues are discussed.

\section{Theoretical framework and basic approaches \\ 2.1 Preliminaries}

We will illustrate the basic approaches for combining column generation and Lagrangian relaxation using the Capacitated Lot-sizing Problem (CLSP). In this problem we determine the timing and level of production for several items on a single machine with limited capacity over a discrete and finite horizon. Let $P$ be the set of products $\{1, \ldots, n\}$ with index $i$ and $T$ the set of time periods $\{1, \ldots, m\}$ with index $t$. We have the following parameters: $d_{i t}$ is the demand of product $i$ in period $t$; $s c_{i}, v c_{i}$ and $h c_{i}$ are the set up cost, variable production cost and holding cost for product $i$, respectively; $v t_{i}$ is the variable production time for product $i$ and $\operatorname{cap}_{t}$ is the capacity in period $t$. There are three decision variables: $x_{i t}$ is the amount of production of product $i$ in period $t ; s_{i t}$ is the inventory level of product $i$ at the end of period $t ; y_{i t}=1$ if there is a set up for product $i$ in period $t, y_{i t}=0$ otherwise. The mathematical formulation of the CLSP is then as follows:

$$
\begin{aligned}
\min \sum_{i \in P} \sum_{t \in T}\left(s c_{i} y_{i t}+v c_{i} x_{i t}+h c_{i} s_{i t}\right) & \\
s_{i, t-1}+x_{i t}=d_{i t}+s_{i t} & \forall i \in P, \forall t \in T, \\
x_{i t} \leq M y_{i t} & \forall i \in P, \forall t \in T, \\
\sum_{i \in P} v t_{i} x_{i t} \leq c a p_{t} & \forall t \in T, \\
y_{i t} \in\{0,1\}, x_{i t} \geq 0, s_{i t} \geq 0, s_{i, 0}=0 & \forall i \in P, \forall t \in T .
\end{aligned}
$$

The objective function (1.1) minimizes the total costs, consisting of the set up cost, the variable production cost and the inventory holding 
cost. Constraints (1.2) are the inventory balancing constraints: inventory left over from the previous period plus current production can be used to satisfy current demand or build up more inventory. Constraints (1.3) are the set up forcing constraints: if there is any positive production in period $t$, a set up is enforced. In order to make the formulation stronger, the ' $\mathrm{big} M$ ' is usually set to the minimum of the sum of the remaining demand over the horizon and the total production which is possible with the available capacity. Next, there is a constraint on the available capacity in each period (1.4). Finally, there are the nonnegativity and integrality constraints (1.5). We let $v_{L S}$ and $\bar{v}_{L S}$ denote the optimal objective value for problem (1.1)-(1.5) and its LP relaxation, respectively.

Decomposition approaches for this problem hinge on the observation that when we disregard the capacity constraints (1.4), the problem decomposes into an uncapacitated lot-sizing problem for each item $i$. Let $S^{i}$ be the set of feasible solution for subproblem $i: S^{i}=\left\{\left(x_{i t}, y_{i t}, s_{i t}\right) \mid(1.2)\right.$, (1.3), (1.5)\} and $S=\bigcup_{i \in P} S^{i}$. In the Dantzig-Wolfe decomposition, we keep the capacity constraints in the master and add a convexity constraint for each item (Manne (1958), Dzielinski and Gomory (1965)). The new columns represent a production plan for a specific item over the full time horizon. Let $Q_{i}$ be the set of all extreme point production plans for item $i ; z_{i j}$ is the new variable representing production plan $j$ for item $i ; c_{i j}$ is the total cost of set up, production and inventory for production plan $j$ for item $i$ and $r_{i j t}$ is the capacity usage of the production in period $t$ according to plan $j$ for item $i$. The LP relaxation of a restricted master problem then looks as follows:

$$
\begin{gathered}
\bar{v}_{R D W L S}=\min \sum_{i \in P} \sum_{j \in \tilde{Q}_{i}} c_{i j} z_{i j} \\
\sum_{i \in P} \sum_{j \in \tilde{Q}_{i}} r_{i j t} z_{i j} \leq c a p_{t} \quad \forall t \in T, \\
\sum_{j \in \tilde{Q}_{i}} z_{i j}=1 \quad \forall i \in P, \\
z_{i j} \geq 0 \quad \forall i \in P, \forall j \in \tilde{Q}_{i} .
\end{gathered}
$$

where $\tilde{Q}_{i}$ is a subset of $Q_{i}$. Additional columns (variables) are generated when they are needed, using the information of the optimal dual variables $\mu_{t}$ and $\pi_{i}$ of the capacity and convexity constraints, respectively. In the pricing problem, we check for each item $i$ if we can generate 
a new column by solving the following subproblem:

$$
r c_{i}^{*}(\mu, \pi)=\min _{(x, y, s) \in S^{i}} \sum_{t \in T}\left(s c_{i} y_{i t}+v c_{i} x_{i t}+h c_{i} s_{i t}\right)+\sum_{t \in T} v t_{i} x_{i t} \mu_{t}-\pi_{i} .
$$

If such a column with negative reduced cost is found, we add it to the restricted master, reoptimize this problem and perform another pricing iteration; otherwise we have found the optimal Dantzig-Wolfe bound, $\bar{v}_{D W L S}$.

In Lagrangian relaxation, the capacity constraint (1.4) is dualized in the objective function with non-negative multipliers $u=\left\{u_{1}, u_{2}, \ldots, u_{m}\right\}$ :

$\bar{v}_{L R L S}(u)=\min _{(x, y, s) \in S} \sum_{i \in P} \sum_{t \in T}\left(s c_{i} y_{i t}+v c_{i} x_{i t}+h c_{i} s_{i t}\right)-\sum_{t \in T} u_{t}\left(\operatorname{cap}_{t}-\sum_{i \in P} v t_{i} x_{i t}\right)$.

The Lagrangian problem also decomposes into single item uncapacitated lot-sizing problems. For each item $i$ we have the following subproblem:

$$
\bar{v}_{L R L S, i}(u)=\min _{(x, y, s) \in S^{i}} \sum_{t \in T}\left(s c_{i} y_{i t}+v c_{i} x_{i t}+h c_{i} s_{i t}\right)+\sum_{t \in T} v t_{i} x_{i t} u_{t} .
$$

We see that the subproblem of calculating the minimum reduced cost (1.10) in the Dantzig-Wolfe decomposition and the subproblem in the Lagrangian relaxation (1.12) are identical, except for a constant in the objective function. Solution of the Lagrangian Dual problem gives the maximum lower bound $\bar{v}_{L D L S}=\max _{u \geq 0} \bar{v}_{L R L S}(u)$. In iterative steps, the multipliers are updated in order to attain this Lagrangian Dual bound. Let $x^{*}=\left(x_{11}^{*}, x_{12}^{*}, \ldots, x_{1 m}^{*}, \ldots, x_{n 1}^{*}, x_{n 2}^{*}, \ldots, x_{n m}^{*}\right)$ be the optimal production quantities for the Lagrangian problem (1.11) with multipliers $u^{k}$ at iteration $k$, then the following standard subgradient update formulas (Fisher (1981)) result in a new set of multipliers $u^{k+1}$ :

$$
\begin{aligned}
u_{t}^{k+1} & =\max \left(0, u_{t}^{k}-\lambda_{k}\left(\operatorname{cap}_{t}-\sum_{i \in P} v t_{i} x_{i t}^{*}\right)\right) \quad t=1, \ldots, m, \\
\lambda_{k} & =\alpha \frac{\left(u b-\bar{v}_{L R L S}\left(u^{k}\right)\right)}{\sum_{t \in T}\left(\operatorname{cap}_{t}-\sum_{i \in P} v t_{i} x_{i t}^{*}\right)^{2}} .
\end{aligned}
$$

Equation (1.14) determines the step-size, where $0<\alpha \leq 2$ and the value $u b$ is an upper bound on $v_{L S}$.

During column generation, the value of the restricted master $\bar{v}_{R D W L S}$ provides an upper bound on the value of the optimal Dantzig-Wolfe relaxation $\bar{v}_{D W L S}$. However, a lower bound can be easily calculated as 
well. Let $r c_{i}^{*}(\mu, \pi)$ be the minimum reduced cost for subproblem $i$ with the current optimal dual variables $\pi$ and $\mu$, then

$$
\sum_{i \in P} r c_{i}^{*}(\mu, \pi)+\bar{v}_{R D W L S} \leq \bar{v}_{D W L S} \leq \bar{v}_{R D W L S} .
$$

This lower bound is actually equal to the Lagrangian lower bound using the current optimal dual variables $\mu$ as multipliers:

$$
\begin{aligned}
\bar{v}_{L R L S}(\mu) & =\sum_{i \in P} \bar{v}_{L R L S, i}(\mu)-\sum_{t \in T} \mu_{t} \operatorname{cap}_{t} \\
& =\sum_{i \in P} \bar{v}_{L R L S, i}(\mu)-\sum_{i \in P} \pi_{i}+\sum_{i \in P} \pi_{i}-\sum_{t \in T} \mu_{t} c a p_{t} \\
& =\sum_{i \in P} r c_{i}^{*}(\mu, \pi)+\bar{v}_{R D W L S},
\end{aligned}
$$

where in the final step, equivalence between $\sum_{i \in P} \pi_{i}-\sum_{t \in T} \mu_{t} c a p_{t}$ and $\bar{v}_{R D W L S}$ follows from LP duality. This lower bound was already proposed by Lasdon and Terjung (1971) who used column generation to solve a large production scheduling problem. It has also been discussed for other specific problems such as discrete Lot-sizing and scheduling (Jans and Degraeve (2004)), machine scheduling (Van den Akker, Hurkens and Savelsbergh (2000)), vehicle routing (Sol (1994)), a multicommodity network-flow problem (Holmberg and Yuan (2003)) and the cutting stock problem (Vanderbeck (1999)). A general discussion can be found in Wolsey (1998) and Martin (1999). Vanderbeck and Wolsey (1996) provide a slight strengthening of this bound. The bound can be used for early termination of the column generation procedure, reducing the tailing-off effect. For IP problems with an integer objective function value, we can also stop if the value of this lower bound rounded up is equal to the value of the restricted master rounded up.

\subsection{Using Lagrangian relaxation on the extended formulation}

Instead of using the simplex algorithm to obtain the optimal dual variables of the (restricted) master problem, one can also use Lagrangian relaxation to approximate these values. Cattrysse et al. (1993) and Jans and Degraeve (2004) apply this technique for solving a variant of the Capacitated Lot-Sizing Problem. A similar integration of Dantzig-Wolfe decomposition and Lagrangian relaxation is also used for the generalized assignment problem (Cattrysse, Salomon and Van Wassenhove (1994)), 
and integrated vehicle and crew scheduling which is the topic of Section 1.3 .

In order to approximately solve the LP relaxation of the restricted master (1.6)-(1.9), we dualize the capacity constraint (1.7) into the objective function (1.6):

$$
\begin{gathered}
\bar{v}_{L R-R D W}(u)=\min \sum_{i \in P} \sum_{j \in \tilde{Q}_{i}} c_{i j} z_{i j}-\sum_{t \in T} u_{t}\left(c a p_{t}-\sum_{i \in P} \sum_{j \in \tilde{Q}_{i}} r_{i j t} z_{i j}\right) \\
\sum_{j \in \tilde{Q}_{i}} z_{i j}=1 \quad \forall i \in P \\
z_{i j} \geq 0 \quad \forall i \in P, \forall j \in \tilde{Q}_{i} .
\end{gathered}
$$

The problem decomposes into subproblems per item that are easy to solve, because taking the column with the lowest total cost for each item results in the optimal solution. The optimal Lagrangian multipliers are iteratively approximated via a standard subgradient optimization procedure. At the end of a subgradient phase, the Lagrangian multipliers $u_{t}$ are an approximation of the optimal dual variables $\mu_{t}$. Next, the optimal dual variable of the convexity constraint for item $i$ can be approximated as follows:

$$
p_{i}=\min _{j \in \tilde{Q}_{i}}\left(c_{i j}+\sum_{t \in T} u_{t} r_{i j t}\right) .
$$

These Lagrangian multipliers can be used to generate new columns in the pricing subproblem (1.10). The new columns are added to the restricted master and in a subsequent step the optimal dual variables $\mu$ and $\pi$ for the updated restricted master are again approximated by Lagrangian relaxation.

Given the Lagrangian multipliers $u_{t}$ and $p_{i}$, we can still compute a lower bound:

$$
\sum_{i \in P} r c_{i}^{*}(u, p)+\bar{v}_{L R-R D W}(u) \leq \bar{v}_{D W L S} .
$$


This can again be proven by starting from the Lagrangian relaxation $\bar{v}_{L R L S}(u)(1.11)$, which gives a valid lower bound for any $u \geq 0$ :

$$
\begin{aligned}
\bar{v}_{L R L S}(u) & =\sum_{i \in P} \bar{v}_{L R L S, i}(u)-\sum_{i \in P} p_{i}+\sum_{i \in P} p_{i}-\sum_{t \in T} u_{t} c a p_{t} \\
& =\sum_{i \in P} r c_{i}^{*}(u, p)+\sum_{i \in P} p_{i}-\sum_{t \in T} u_{t} c a p_{t} \\
& =\sum_{i \in P} r c_{i}^{*}(u, p)+\sum_{i \in P} \min _{j \in \tilde{Q}_{i}}\left(c_{i j}+\sum_{t \in T} u_{t} r_{i j t}\right)-\sum_{t \in T} u_{t} c a p_{t} \\
& =\sum_{i \in P} r c_{i}^{*}(u, p)+\bar{v}_{L R-R D W}(u) .
\end{aligned}
$$

What are the advantages of approximating the optimal dual variables by Lagrangian relaxation instead of computing them exactly with a simplex algorithm? Bixby et al. (1992) and Barnhart et al. (1998) note that in case of alternative dual solutions, column generation algorithms seem to work better with dual variables produced by interior point methods than with dual variables computed with simplex algorithms. The latter give a vertex of the face of solutions whereas interior point algorithms give a point in the center of the face, providing a better representation of it. From that perspective, Lagrangian multipliers may also provide a better representation and speed up convergence. Computational experiments from Jans and Degraeve (2004) indicate that using Lagrangian multipliers indeed speeds up convergence and decreases the problem of degeneracy. Lagrangian relaxation has the additional advantage that during the subgradient phase possibly feasible solutions are generated. The subgradient updating is also fast and easy to implement. Finally, this procedure eliminates the need for a commercial LP optimizer.

\subsection{Using Lagrangian relaxation on the compact formulation}

This approach is based on the observation that when the Lagrangian relaxation is obtained by dualizing exactly those constraints that are the linking constraints in the Dantzig-Wolfe reformulation, the same subproblem results. Consequently, the solutions generated by the Lagrangian subproblems can also be added as new columns to the master. This was first proposed by Barahona and Jensen (1998) for a plant location problem and by Degraeve and Peeters (2003) for the cutting stock problem. These applications are discussed in Sections 1.4 and 1.5, respectively. It has also been applied successfully to the Capacitated 
Lot-sizing Problem (Degraeve and Jans (2003)), that is used again to illustrate the technique. The procedure essentially consists of a nested double loop. In the outer loop, optimal dual variables for the restricted master problem (1.6)-(1.9) are obtained by the simplex method. In the inner loop, the Lagrangian subproblem of the compact formulation (1.11) is solved during several iterations, each time with dual variables which are updated with a subgradient optimization procedure. A generic procedure is depicted in Figure 1.1.

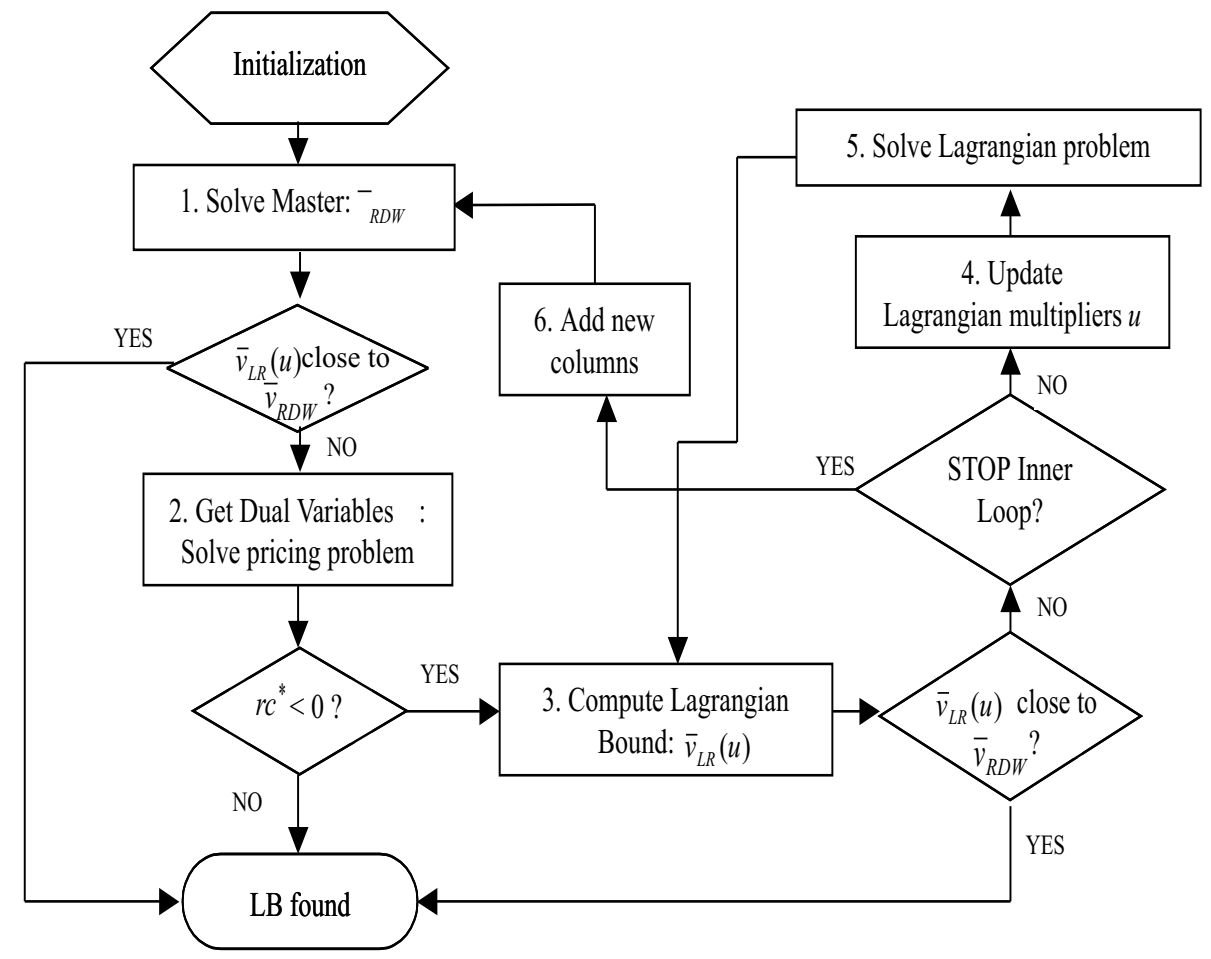

Figure 1.1. Outline of algorithm

After initialization, the LP relaxation of the restricted master (1.6)(1.9) is solved (Box 1). Next the optimal dual variables $\mu$ and $\pi$ are passed to the pricing problem (1.10), which is then solved to find a new column (Box 2). If the reduced cost is non-negative for each subproblem, then the Dantzig-Wolfe bound $\bar{v}_{D W}$ is found. Otherwise, the inner loop starts (Box 3), where in the first iteration the Lagrangian bound $\bar{v}_{L R}(\mu)$ (1.11) is computed, using the optimal dual variables of the restricted master. This bound is then compared with the objective value of the restricted master $\bar{v}_{R D W}$. For a pure integer programming problem with 
integer coefficients in the objective function, the procedure terminates if both values rounded up are equal, and the Dantzig-Wolfe bound equals $\left\lceil\bar{v}_{R D W}\right\rceil=\left\lceil\bar{v}_{L R}(u)\right\rceil$. For a mixed integer programming problem, the algorithm may be terminated, if the difference between both values is smaller than a pre-specified percentage. Other stopping criteria could also be checked. For instance, Barahona and Jensen (1998) stop the inner loop after a fixed number of iterations. If no stopping criteria are satisfied, then the Lagrangian multipliers are updated using subgradient optimization (Box 4). The value $u b$ in (1.14) is an upper bound on $\bar{v}_{L D}$, and therefore, $u b$ can be set equal to the LP bound of the last solved restricted master $\bar{v}_{R D W}$, since $\bar{v}_{R D W} \geq \bar{v}_{D W}=\bar{v}_{L D}$. Next the algorithm proceeds with solving a new Lagrangian problem, with the updated multipliers (Box 5). The Lagrangian bound is computed again and the inner loop continues, until a stopping criterion is met. Next, we switch back to the outer loop. We add to the restricted master the columns, which were generated in the inner loop if they are not yet present (Box 6).

The main advantage of this procedure is that the LP relaxation of the master problem does not need to be solved each time to get new dual variables necessary for pricing out a new column. Solving the LP relaxation to optimality is computationally much more expensive than performing an iteration of the subgradient optimization procedure. At each subgradient iteration, a new column is found and these columns are expected to be "good" because the Lagrangian multipliers prices converge towards the optimal dual variables of the LP relaxation of the restricted master. A second advantage is that we can stop the column generation short of proving LP optimality of the master, because the Lagrangian relaxation provides lower bounds on the optimal LP value. Barahona and Jensen (1998) mention this fact as the main motivation for performing a number of subgradient iterations between two consecutive outer loop iterations. This procedure tries to combine the speed of subgradient optimization with the exactness of the Dantzig-Wolfe algorithm. In addition, the procedure provides a primal solution on which branching decisions or rounding heuristics can be based, which is not case if only subgradient optimization is used. Computational results from Degraeve and Jans (2003) indicate that this method speeds up the column generation procedure. With this hybrid method, it takes about half the time to find the lower bound compared to the traditional method. 


\section{Application 1: Integrated Vehicle and Crew Scheduling}

In this section we discuss the application of a combined column generation/Lagrangian relaxation algorithm to the integrated vehicle and crew scheduling problem. Vehicle and crew scheduling are two of the most important planning problems in a bus company. Since this is the topic of Chapter ?? by Borndörfer et al., we will not discuss these problems themselves in detail. Here we only present a formulation for the integrated problem (in case of multiple-depots) to which we apply the approach outlined in Subsection 1.2.2. Some interesting, recent references on the integrated problem are Freling (1997), Haase, Desaulniers and Desrosiers (2001) and Freling, Huisman and Wagelmans (2003) for the single-depot case, and Gaffi and Nonato (1999) and Huisman, Freling and Wagelmans (2003) for the multiple-depot case.

\subsection{Mathematical Formulation}

Let $N=\{1,2, \ldots, n\}$ be the set of trips, numbered according to increasing starting time. Define $D$ as the set of depots and let $s^{d}$ and $t^{d}$ both represent depot $d$. Furthermore, for the crew we distinguish two types of tasks, viz., trip tasks corresponding to trips, and dh-tasks corresponding to deadheading. A deadhead is defined as a period that a vehicle is moving in time or space without passengers. $E^{d}$ is the set of deadheads between two trips $i$ and $j$.

We define the vehicle scheduling network $G^{d}=\left(V^{d}, A^{d}\right)$, which is an acyclic directed network with nodes $V^{d}=N^{d} \cup\left\{s^{d}, t^{d}\right\}$, and arcs $A^{d}=E^{d} \cup\left(s^{d} \times N^{d}\right) \cup\left(N^{d} \times t^{d}\right)$. Note that $N^{d}$ is the part of $N$ corresponding to depot $d$, since it is not necessary that all trips can be served from each depot. Let $c_{i j}^{d}$ be the vehicle cost of $\operatorname{arc}(i, j) \in A^{d}$.

Furthermore, let $K^{d}$ denote the set of duties corresponding to depot $d$ and $f_{k}^{d}$ denote the crew cost of duty $k \in K^{d}$, respectively. Moreover, $K^{d}(i)$ denotes the set of duties covering the trip task corresponding to trip $i \in N^{d}$, which means that we assume that a trip corresponds to exactly one task. $K^{d}(i, j)$ denotes the set of duties covering the dh-tasks corresponding to deadhead $(i, j) \in A^{d}$. Decision variable $y_{i j}^{d}$ indicates whether an arc $(i, j)$ is used and assigned to depot $d$ or not, while $x_{k}^{d}$ indicates whether duty $k$ corresponding to depot $d$ is selected in the solution or not. The multiple-depot vehicle and crew scheduling problem (MD-VCSP) can be formulated as follows. 


$$
\begin{gathered}
\min \sum_{d \in D} \sum_{(i, j) \in A^{d}} c_{i j}^{d} y_{i j}^{d}+\sum_{d \in D} \sum_{k \in K^{d}} f_{k}^{d} x_{k}^{d} \\
\sum_{d \in D} \sum_{j:(i, j) \in A^{d}} y_{i j}^{d}=1 \quad \forall i \in N, \\
\sum_{d \in D} \sum_{i:(i, j) \in A^{d}} y_{i j}^{d}=1 \quad \forall j \in N, \\
\sum_{i:(i, j) \in A^{d}} y_{i j}^{d}-\sum_{i:(j, i) \in A^{d}} y_{j i}^{d}=0 \quad \forall d \in D, \forall j \in N^{d}, \\
\sum_{k \in K^{d}(i)} x_{k}^{d}-\sum_{j:(i, j) \in A^{d}} y_{i j}^{d}=0 \quad \forall d \in D, \forall i \in N^{d}, \\
\sum_{k \in K^{d}(i, j)} x_{k}^{d}-y_{i j}^{d}=0 \quad \forall d \in D, \forall(i, j) \in A^{d}, \\
x_{k}^{d} \in\{0,1\} \quad \forall d \in D, \forall k \in K^{d}, \\
y_{i j}^{d} \in\{0,1\} \quad \forall d \in D, \forall(i, j) \in A^{d} .
\end{gathered}
$$

The objective is to minimize the sum of vehicle and crew costs. The first three sets of constraints, (1.22)-(1.24), correspond to the formulation of the vehicle scheduling problem. Constraints (1.25) assure that each trip task will be covered by a duty from a depot if and only if the corresponding trip is assigned to this depot. Furthermore, constraints (1.26) guarantee the link between vehicles and crews. That is, a vehicle performs deadhead $(i, j)$ if and only if the corresponding dh-task is assigned to a driver from the same depot.

Notice that this formulation is already an extended one. We would obtain a similar formulation, if we would apply Dantzig-Wolfe decomposition on a compact formulation of this problem (see e.g. Desrosiers et al. (1995) how this transformation can be applied on the multicommodity flow problem with resource constraints, which has as special case all kind of vehicle and crew scheduling problems).

\subsection{Algorithm}

Below we first give a schematic overview of a combined column generation/Lagrangian relaxation algorithm to solve the MD-VCSP. Afterwards, we discuss the steps related to Lagrangian relaxation (1,2 and 4) in more detail. For details about the other steps, we refer to Huisman, Freling and Wagelmans (2003). 
Step 0. Find an initial feasible solution and take as initial set of columns the duties in that solution.

Step 1. Solve a Lagrangian dual problem with the current set of columns approximately, i.e. perform some subgradient optimization steps to update the multipliers. This gives a lower bound for the current restricted master.

Step 2. Modify multipliers to prevent that columns are generated twice.

Step 3. Generate columns (duties) with negative reduced cost and update the set of columns.

Step 4. Compute an estimate of a lower bound for the (full) master problem. If the gap between this estimate and the lower bound found in Step 1 is small enough (or another termination criterion is satisfied), go to Step 5; otherwise, return to Step 1.

Step 5. Construct feasible solutions by applying a Lagrangian heuristic.

To approximate the optimal value of the restricted master problem in Step 1, we use the relaxation of model MD-VCSP, where the equality signs in the constraints (1.24)-(1.26) are first replaced by "greaterthan-or-equal" signs. These constraints are subsequently relaxed in a Lagrangian way. That is, we associate non-negative Lagrangian multipliers $\kappa_{j}^{d}, \lambda_{i}^{d}, \mu_{i j}^{d}$ with constraints (1.24), (1.25), (1.26), respectively. Then the optimal solution of the remaining Lagrangian subproblem can be obtained by inspection for the $x$ variables and by solving a large single-depot vehicle scheduling problem (SDVSP) for the $y$ variables.

The values of the Lagrangian multipliers obtained after applying a subgradient algorithm can be used to generate new columns. However, to assure that all columns in the current restricted master problem have non-negative reduced costs such that the corresponding duties will not be generated again in the pricing problem, we use an additional procedure (Step 2) to update the Lagrangian multipliers after solving the Lagrangian relaxation. This can be done with a greedy heuristic, that modifies these multipliers in such a way that columns in the current restricted master problem $\tilde{K}^{d}$ have non-negative reduced costs and the value of the Lagrangian function does not decrease. We denote $\bar{f}_{k}^{d}$ as the reduced costs of column $k \in K^{d}$, which is equal to

$$
f_{k}^{d}-\sum_{i \in N(k, d)} \lambda_{i}^{d}-\sum_{(i, j) \in A(k, d)} \mu_{i j}^{d}
$$


where $N(k, d)$ and $A(k, d)$ are the set of trip tasks and dh-tasks in duty $k$ from depot $d$, respectively. The heuristic is described below (see also Freling (1997) and Carraresi, Girardi and Nonato (1995)):

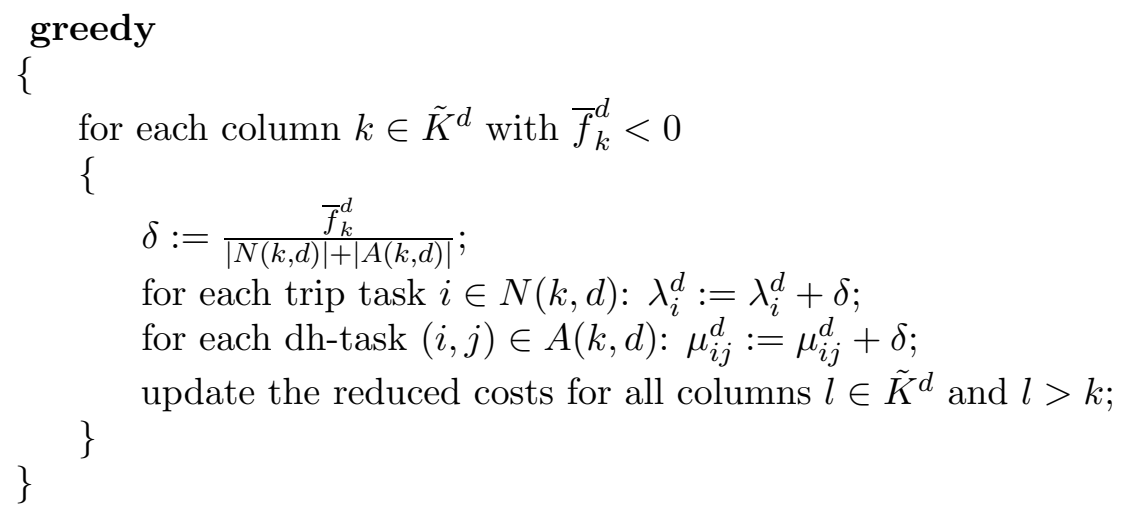

Finally, we will discuss Step 4, where we compute an estimate of a lower bound for the master problem given a lower bound for the current restricted master. The latter bound, denoted by $\Phi^{\prime}(\kappa, \lambda, \mu)$, is obtained in Step 1. Then the expression:

$$
\Phi^{\prime}(\kappa, \lambda, \mu)+\sum_{d \in D} \sum_{k \in K^{d} \backslash \tilde{K}^{d}} \min \left(\bar{f}_{k}^{d}, 0\right)
$$

is a lower bound for the (full) master problem for each vector $(\kappa, \lambda, \mu)$. This can be proven in a similar way as in Subsection 1.2.2. Therefore, we will skip this proof here.

Notice, however, that we do not calculate this lower bound in each iteration, since for generating new columns it is not necessary to calculate the reduced costs for all of them. Therefore, we estimate this bound in each iteration by taking only into account the reduced costs of the columns that we actually add to the master problem. This estimate can be used to stop the column generation part of the algorithm earlier without exactly obtaining a lower bound.

\subsection{Some results}

The algorithm presented in the previous subsection has been used to solve several problem instances arising from real-world applications as well as randomly generated instances. In Table 1.1 we summarize some of the results for randomly generated instances with two depots (see Huisman, Freling and Wagelmans (2003)). We denote the average 
number of iterations of the column generation algorithm, and the average computation times for the master problem (cpu m.) and pricing problem (cpu p.), respectively. Furthermore, we give the total average computation time for computing the lower bound (cpu t.). These averages are computed over the instances for which a lower bound is found within 3 hours of cpu time on a Pentium III $450 \mathrm{MHz}$ personal computer (128MB RAM). Therefore, we also denote the number of instances (out of 10) for which we actually found a lower bound. In the remainder of the table, we denote the average gaps between the lower and upper bounds.

Table 1.1. Computational Results MD-VCSP.

\begin{tabular}{ccccc}
\hline \# trips & 80 & 100 & 160 & 200 \\
\hline \# iter. & 17.4 & 25.2 & 36.8 & 39.5 \\
cpu m. & 154.7 & 403.9 & 982.8 & 1641.5 \\
cpu p. & 148.7 & 510.7 & 3529.8 & 4769.5 \\
cpu t. & 317.5 & 942.3 & 4721.3 & 6675.0 \\
\# found & 10 & 10 & 4 & 2 \\
\hline gap (\%) & 5.37 & 5.31 & 5.75 & 6.52 \\
\hline
\end{tabular}

In Table 1.1, we only provide results for instances up to 200 trips, since for larger instances we were not able to compute a lower bound within 3 hours computation time. The average gaps between the feasible solutions and the lower bound are about $5 \%$ for those instances. However, for large instances we can still use the suggested algorithm to compute feasible solutions by terminating the lower bound phase after a maximum computation time and then continue with Step 5. In practice, this is already quite satisfactory. Therefore, these types of algorithms can be used to solve practical problem instances in an integrated way.

\section{Application 2: Plant Location}

Barahona and Jensen (1998) apply the procedure described in Subsection 1.2.3 to a plant location problem with minimum inventory. Given a set $N$ of customers, each requiring a set of parts $D_{i} \subset P, i \in N$, where $P$ denotes the set of all parts, and a set of $M$ possible locations, the objective is to minimize the total costs such that every customer is served, a bound on the total number of warehouses is not exceeded and a service criterion is met. The total costs consist of a fixed costs $f_{j}$, for $j \in M$, if a warehouse is opened at location $j$, a transportation $\operatorname{cost} c_{i j}$ if customer $i$ is served from warehouse $j$, and an inventory cost $h_{j k}$, if part $k$ is stored 
in warehouse $j$. A part must be stored in a warehouse if a customer, requiring that part, is assigned to the warehouse. The service criterion implies that a given percentage of the total demand must be delivered within a certain time limit. Let $y_{j}$ be 1 , if warehouse $j$ is opened, and 0 otherwise, let $x_{i j}$ be 1 if customer $i$ is assigned to warehouse $j$, and 0 otherwise, and let $z_{j k}$ be 1 , if part $k$ must be stored in warehouse $j$, and 0 otherwise, then the model can be stated as follows.

$$
\begin{array}{rlrl}
\min \sum_{j \in M} f_{j} y_{j} & +\sum_{i \in N} \sum_{j \in M} c_{i j} x_{i j}+\sum_{j \in M} \sum_{k \in P} h_{j k} z_{j k} \\
\sum_{j \in M} x_{i j} & =1 \quad \forall i \in N, & \\
\sum_{i \in N} \sum_{j \in M} d_{i j} x_{i j} & \geq t, & \\
\sum_{j \in M} y_{j} \leq L, & \\
x_{i j} \leq y_{j} & \forall i \in N, \forall j \in M, \\
x_{i j} \leq z_{j k} & \forall i \in N, \forall j \in M, \forall k \in D_{i}, \\
x_{i j}, y_{j}, z_{j k} & \in\{0,1\} \quad \forall i \in N, \forall j \in M, \forall k \in P,
\end{array}
$$

Constraints (1.32) impose that every customer must be assigned to one location. Constraint (1.33) is the service criterion, i.e. suppose that the company would like that $95 \%$ of the demand can be served within two hours, then $t$ equals $95 \%$ of the total demand and $d_{i j}$ is equal to the demand of customer $i$, if the travel time between $i$ and $j$ is less than two hours, and 0 otherwise. Constraint (1.34) implies that at most $L$ locations can be opened. Constraints (1.35) and (1.36) define the relations between the variables, i.e. a customer can only be assigned to a warehouse, if the warehouse is open (1.35), and, if customer $i$ is assigned to a warehouse, then all parts $D_{i}$ of customer $i$ must be present in the warehouse (1.36).

The Dantzig-Wolfe reformulation consists of implicitly considering every possible assignment of customers to locations. Hence, the objective function and constraints of (the LP relaxation of) the master problem correspond to (1.31)-(1.34) and the original variables are replaced by a convex combination of the extreme points of the polytope defined by (1.35)-(1.37). Barahona and Jensen (1998) show the pricing problem is equivalent to a minimum cut problem. They observed that the convergence of the Dantzig-Wolfe algorithm is very slow for this problem and that the lower bound obtained by adding the reduced cost of the 
columns that price out to the value of the current restricted master, is very poor in the first iterations of the Dantzig-Wolfe algorithm and improves only slowly. After solving the LP relaxation of the current restricted master, they perform a fixed number of subgradient iterations on the original problem to improve the bound, using the masters' optimal dual variables as starting values for the subgradient procedure. Next, all columns are added to the LP relaxation of the restricted master, which is then re-optimized. If the new optimal objective value and the Lagrangian lower bound are close to each other, then a heuristic is applied to obtain an integer solution. They are able to obtain good solutions for problems with about 200 locations, 200 parts and 200 customers within about one hour of computation time on a RS6000-410, using OSL (IBM Corp. (1995)) to solve the LPs.

\section{Application 3: Cutting Stock}

Degraeve and Peeters (2000) use a combination of the simplex method and subgradient optimization to speed up the convergence of the column generation algorithm of Gilmore and Gomory (1961) for the onedimensional cutting stock problem (CSP). This procedure is used to compute the LP relaxation at every node of the branch-and-price tree of the algorithm described in Degraeve and Peeters (2003). The CSP can be defined as follows. Given an unlimited stock of a raw material type of length $c$ and a set of $n$ items with widths $w_{1}, \ldots, w_{n}$ and demands $d_{1}, \ldots, d_{n}$, cut as few raw material types as possible, such that the demand is satisfied and the total width of the items cut from a raw material type does not exceed its length $c$. Let $P$ be the set of all feasible cutting patterns, or

$$
P=\left\{p \in \mathbb{Z}_{+}^{n}: \sum_{i=1}^{n} w_{i} p_{i} \leq c\right\} .
$$

Let $z_{p}$ be the number of times pattern $p$ is selected in the solution, then the Gilmore and Gomory formulation can be stated as follows:

$$
\begin{aligned}
& \min \sum_{p \in P} z_{p} \\
& \sum_{p \in P} p_{i} z_{p} \geq d_{i} \quad \forall i \in 1, \ldots, n, \\
& z_{p} \in\{0,1,2, \ldots\} \quad \forall p \in P .
\end{aligned}
$$

The objective function (1.39) minimizes the total number of cut raw material, whereas constraints (1.40) are the demand constraints and 
constraints (1.41) the integrality and non-negativity restrictions. The LP relaxation of (1.39)-(1.41) can be solved by column generation, where the pricing problem is a bounded knapsack problem, if one does not allow that the number of items present in a cutting pattern exceeds the demand, i.e. $p_{i} \leq d_{i}$.

Using the procedure described in Subsection 1.2.3, Degraeve and Peeters (2000) are able to achieve a substantial reduction in required CPU time to solve the LP relaxation of (1.39)-(1.41). Like Barahona and Jensen (1998), they use a limit on the number of subgradient iterations in the inner loop of Figure 1.1, but, in addition, the inner loop is interrupted, if a new column has non-negative reduced cost, or if the Lagrangian bound rounded up equals the master objective value rounded up, as explained earlier in Figure 1.1. If this last condition holds, the Dantzig-Wolfe lower bound is found. Otherwise, all different columns generated in the inner loop are added to the restricted master. First it is checked if the value of the best Lagrangian lower bound rounded up is equal to the value of the new restricted master rounded up. Then, the algorithm can be terminated, otherwise the next iteration of the outer loop continues.

Table 1.2 presents the results for cutting stock instances with 50, 75 and 100 items for 4 different width intervals given in the first row, in which the item widths are uniformly distributed. The demand is uniformly distributed with an average of 50 and the raw material length equals 10000. The experiments were run on a Dell Pentium Pro 200Mhz PC (Dell Dimension XPS Pro 200n) using the Windows95 operating system, the computation times are averages over 20 randomly drawn instances and given in seconds. The LPs are solved using the industrial LINDO optimization library version 5.3 (Schrage (1995)). The columns labelled "DW" present the traditional Dantzig-Wolfe algorithm and the columns labelled "CP" present the results of the combined procedure of Figure 1.1. We observe that the reduction in CPU time is higher, when the number of items is higher, and can be as high as a factor 8 .

Table 1.2. Computational results, Cutting Stock Problem.

\begin{tabular}{ccccccccc}
\hline int & \multicolumn{2}{c}{$[1,2500]$} & \multicolumn{2}{c}{$[1,5000]$} & \multicolumn{2}{c}{$[1,7500]$} & \multicolumn{2}{c}{$[1,10000]$} \\
$n$ & $D W$ & $C P$ & $D W$ & $C P$ & $D W$ & $C P$ & $D W$ & $C P$ \\
\hline 50 & 0.44 & 0.21 & 1.47 & 0.52 & 0.67 & 0.46 & 0.14 & 0.10 \\
75 & 1.14 & 0.47 & 4.82 & 1.12 & 4.26 & 1.14 & 0.53 & 0.27 \\
100 & 3.19 & 0.84 & 15.96 & 2.05 & 14.78 & 3.99 & 1.65 & 0.73 \\
\hline
\end{tabular}




\section{Conclusion}

We discussed two ways to combine Lagrangian relaxation and column generation. Since this combination has not been used quite often, there are many interesting research questions open. For example, should we use another method to approximate the Lagrangian Dual, e.g. a multiplier adjustment method? Furthermore, when implementing such algorithms one has to make decisions with respect to issues such as column management.

In the first method, we used Lagrangian relaxation to solve the extended formulation. Therefore, no simplex method was necessary anymore, which has several advantages. First of all, it decreases the problem of degeneracy and speeds up the convergence. Furthermore, master problems with a larger number of constraints are most often faster solved with Lagrangian relaxation than with a LP solver. We showed this by solving the multiple-depot vehicle and crew scheduling problem.

In the second method, Lagrangian relaxation was used to generate new columns. It is an effective method to speed up convergence of the Dantzig-Wolfe column generation algorithm. The method seems to be quite robust, since it gives good results on three totally different problems, and this without much fine-tuning of the parameters. Several issues can be further investigated. For example, how many subgradient iterations do we allow in the inner loop of Figure 1.1? This is also related to the number of columns that we want to add in an inner loop: all new columns, the ones with negative reduced cost or only the ones with the most negative reduced cost? Adding more columns leads possibly to a faster convergence, but larger restricted masters are also more difficult to solve. Do we initialize the multipliers in the Lagrangian relaxation part with the best Lagrangian multipliers of the previous step, with the optimal dual variables provided by the simplex algorithm for the current restricted master, or some combination? Clearly, there are ample opportunities for research into the effective combination of column generation and Lagrangian relaxation.

\section{References}

F. Barahona and R. Anbil. The volume algorithm: producing primal solutions with a subgradient method. Mathematical Programming Series A, 87:385-399, 2000.

F. Barahona and D. Jensen. Plant location with minimum inventory. Mathematical Programming, 83:101-112, 1998. 
C. Barnhart, E.L. Johnson, G.L. Nemhauser, M.W.P. Savelsbergh and P.H. Vance. Branch-and-price: Column generation for solving huge integer programs. Operations Research, 46:316-329, 1998.

R.E. Bixby, J.W. Gregory, I.J. Lustig, R.E. Marsten, and D.F. Shanno. Very large-scale linear programming: a case study in combining interior point and simplex methods. Operations Research, 40:885-897, 1992.

P. Carraresi, L. Girardi, and M. Nonato. Network models, Lagrangean relaxation and subgradients bundle approach in crew scheduling problems. In: J.R. Daduna, I. Branco, and J.M. P. Paixão (eds), ComputerAided Transit Scheduling, Proceedings of the Sixth International Workshop, pp. 188-212. Springer Verlag, 1995.

D.G. Cattrysse, M. Salomon, R. Kuik and L.N. Van Wassenhove. A dual ascent and column generation heuristic for the discrete lotsizing and scheduling problem with setup times. Management Science, 39:477486, 1993.

D.G. Cattrysse, M. Salomon and L.N. Van Wassenhove. A set partitioning heuristic for the generalized assignment problem. European Journal of Operational Research, 72:167-174, 1994.

G.B. Dantzig and P. Wolfe. Decomposition principles for linear programming. Operations Research, 8:101-111, 1960.

Z. Degraeve and R. Jans. A new Dantzig-Wolfe reformulation and Branchand-Price algorithm for the capacitated lot sizing problem with set up times. Technical Report ERS-2003-010-LIS, ERIM, Erasmus University Rotterdam, the Netherlands, 2003.

Z. Degraeve and M. Peeters. Solving the linear programming relaxation of cutting and packing problems: a hybrid simplex method/ subgradient optimization procedure. Technical Report OR0017, Departement Toegepaste Economische Wetenschappen, K.U. Leuven, Belgium, 2000.

Z. Degraeve and M. Peeters. Optimal integer solutions to industrial cutting-stock problems: Part 2, benchmark results. INFORMS Journal on Computing, 15:58-81, 2003.

J. Desrosiers, Y. Dumas, M.M. Solomon and F. Soumis. Time Constrained Routing and Scheduling. In M.O. Ball, T.L. Magnanti, C.L. Monma, G.L. Nemhauser, (eds), Network Routing, Handbooks in Operations Research and Management Science, vol. 8, pp. 35-139. NorthHolland, 1995.

B.P. Dzielinski and R.E. Gomory. Optimal programming of lot sizes, inventory and labour allocations. Management Science, 11:874-890, 1965.

M. Fischetti and P. Toth. A polyhedral approach to the assymetric traveling salesman problem. Management Science, 43:1520-1536, 1997. 
M.L. Fisher. The lagrangian relaxation method for solving integer programming problems. Management Science, 27:1-18, 1981.

M.L. Fisher. An applications oriented guide to lagrangian relaxation. Interfaces, 15:10-21, 1985.

R. Freling. Models and Techniques for Integrating Vehicle and Crew Scheduling. PhD thesis, Tinbergen Institute, Erasmus University Rotterdam, 1997.

R. Freling, D. Huisman, and A.P.M. Wagelmans. Models and algorithms for integration of vehicle and crew scheduling. Journal of Scheduling, 6:63-85, 2003.

A. Gaffi and M. Nonato. An integrated approach to extra-urban crew and vehicle scheduling. In N.H.M. Wilson, (ed), Computer-Aided Transit Scheduling, pp. 103-128. Springer Verlag, 1999.

A.M. Geoffrion. Lagrangean relaxation for integer programming. Mathematical Programming Study, 2:82-114, 1974.

P.C. Gilmore and R.E. Gomory. A linear programming approach to the cutting stock problem. Operations Research, 9:849-859, 1961.

K. Haase, G. Desaulniers, and J. Desrosiers. Simultaneous vehicle and crew scheduling in urban mass transit systems. Transportation Science, 35:286-303, 2001.

K. Holmberg and D. Yuan. A multicommodity network flow problem with side constraints on paths solved by column generation. INFORMS Journal on Computing, 15:42-57, 2003.

D. Huisman, R. Freling and A.P.M. Wagelmans. Multiple-depot integrated vehicle and crew scheduling. Technical Report EI2003-02, Econometric Institute, Erasmus University Rotterdam, the Netherlands, 2003. To appear in Transportation Science.

IBM Corp. Optimization subroutine library: Guide and references. 1995.

R. Jans and Z. Degraeve. An industrial extension of the discrete lot sizing and scheduling problem. IIE Transactions, 36:47-58, 2004.

L.S. Lasdon and R.C. Terjung. An efficient algorithm for multi-item scheduling. Operations Research, 19:946-969, 1971.

C. Lemaréchal, A.S. Nemirovskii and Y.E. Nesterov. New variants of bundle methods. Mathematical Programming, 69:111-148, 1995.

A. Löbel. Vehicle scheduling in public transit and Lagrangian pricing. Management Science, 44:1637-1649, 1998.

T.L. Magnanti, J.F. Shapiro and M.H. Wagner. Generalized linear programming solves the dual. Management Science, 22:1195-1203, 1976.

A.S. Manne. Programming of economic lot sizes. Management Science, 4:115-135, 1958.

R.K. Martin. Large Scale Linear and Integer Optimization: A Unified Approach. Kluwer Academic Publishers, 1999. 
L. Schrage. LINDO: Optimization software for Linear Programming. Lindo Systems Inc., Chicago IL, 1995.

M. Sol. Column generation techniques for pickup and delivery problems. $\mathrm{PhD}$ thesis, Eindhoven University of Technology, 1994.

J.M. Van den Akker, C.A.J. Hurkens, and M.W.P. Savelsbergh. Timeindexed formulations for machine scheduling problems: Column generation. INFORMS Journal on Computing, 12:111-124, 2000.

F. Vanderbeck. Computational study of a column generation algorithm for bin packing and cutting stock problems. Mathematical Programming Series A, 86:565-594, 1999.

F. Vanderbeck and L.A. Wolsey. An exact algorithm for IP column generation. Operations Research Letters, 19:151-159, 1996.

L.A. Wolsey. Integer Programming. Wiley, 1998. 


\section{Publications in the Report Series Research* in Management}

ERIM Research Program: "Business Processes, Logistics and Information Systems"

2003

Project Selection Directed By Intellectual Capital Scorecards

Hennie Daniels and Bram de Jonge

ERS-2003-001-LIS

http://hdl.handle.net/1765/265

Combining expert knowledge and databases for risk management

Hennie Daniels and Han van Dissel

ERS-2003-002-LIS

http://hdl.handle.net/1765/266

Recursive Approximation of the High Dimensional max Function

Ş. II. Birbil, S.-C. Fang, J.B.G. Frenk and S. Zhang

ERS-2003-003-LIS

http://hdl.handle.net/1765/267

Auctioning Bulk Mobile Messages

S.Meij, L-F.Pau, E.van Heck

ERS-2003-006-LIS

http://hdl.handle.net/1765/274

Induction of Ordinal Decision Trees: An MCDA Approach

Jan C. Bioch, Viara Popova

ERS-2003-008-LIS

http://hdl.handle.net/1765/271

A New Dantzig-Wolfe Reformulation And Branch-And-Price Algorithm For The Capacitated Lot Sizing Problem With Set Up Times

Zeger Degraeve, Raf Jans

ERS-2003-010-LIS

http://hdl.handle.net/1765/275

Reverse Logistics - a review of case studies

Marisa P. de Brito, Rommert Dekker, Simme D.P. Flapper

ERS-2003-012-LIS

http://hdl.handle.net/1765/277

Product Return Handling: decision-making and quantitative support

Marisa P. de Brito, M. (René) B. M. de Koster

ERS-2003-013-LIS

http://hdl.handle.net/1765/278

\footnotetext{
A complete overview of the ERIM Report Series Research in Management: $\underline{\text { http://www.erim.eur.nl }}$

ERIM Research Programs:

LIS Business Processes, Logistics and Information Systems

ORG Organizing for Performance

MKT Marketing

F\&A Finance and Accounting

STR Strategy and Entrepreneurship
} 
Managing Product Returns: The Role of Forecasting

Beril Toktay, Erwin A. van der Laan, Marisa P. de Brito

ERS-2003-023-LIS

http://hdl.handle.net/1765/316

Improved Lower Bounds For The Capacitated Lot Sizing Problem With Set Up Times

Zeger Degraeve, Raf Jans

ERS-2003-026-LIS

http://hdl.handle.net/1765/326

In Chains? Automotive Suppliers and Their Product Development Activities

Fredrik von Corswant, Finn Wynstra, Martin Wetzels

ERS-2003-027-LIS

http://hdl.handle.net/1765/363

Mathematical models for planning support

Leo G. Kroon, Rob A. Zuidwijk

ERS-2003-032-LIS

http://hdl.handle.net/1765/332

How and why communications industry suppliers get "squeezed out" now, and the next phase

L-F Pau

ERS-2003-033-LIS

http://hdl.handle.net/1765/317

Financial Markets Analysis by Probabilistic Fuzzy Modelling

Jan van den Berg, Uzay Kaymak, Willem-Max van den Bergh

ERS-2003-036-LIS

http://hdl.handle.net/1765/323

WLAN Hot Spot services for the automotive and oil industries :a business analysis or : "Refuel the car with petrol and information , both ways at the gas station "

L-F Pau, M.H.P.Oremus

ERS-2003-039-LIS

http://hdl.handle.net/1765/318

A Lotting Method for Electronic Reverse Auctions

U. Kaymak, J.P. Verkade and H.A.B. te Braake

ERS-2003-042-LIS

http://hdl.handle.net/1765/337

Supply Chain Optimisation in Animal Husbandry

J.M. Bloemhof, C.M. Smeets, J.A.E.E. van Nunen

ERS-2003-043-LIS

http://hdl.handle.net/1765/353

A Framework for Reverse Logistics

Marisa P. de Brito and Rommert Dekker

ERS-2003-045-LIS

http://hdl.handle.net/1765/354

An assessment system for rating scientific journals in the field of ergonomics and human factors Jan Dul and Waldemar Karwowski

ERS-2003-048-LIS

http://hdl.handle.net/1765/432 
Circulation of Railway Rolling Stock: A Branch-and-Price Approach

Marc Peeters and Leo Kroon

ERS-2003-055-LIS

http://hdl.handle.net/1765/902

Emerging Multiple Issue e-Auctions

Jeffrey E. Teich, Hannele Wallenius, Jyrki Wallenius and Otto R. Koppius

ERS-2003-058-LIS

http://hdl.handle.net/1765/922

Inventory Management with product returns: the value of information

Marisa P. de Brito and E. A. van der Laan

ERS-2003-060-LIS

http://hdl.handle.net/1765/925

Promising Areas for Future Research on Reverse Logistics: an exploratory study

Marisa P. de Brito

ERS-2003-061-LIS

http://hdl.handle.net/1765/926

A Polynomial Time Algorithm for a Deterministis Joint Pricing and Inventory Model

Wilco van den Heuvel and Albert P.M. Wagelmans

ERS-2003-065-LIS

http://hdl.handle.net/1765/929

A geometric algorithm to solve the ni/g/ni/nd capacitated lot-sizing problem in o(t²) time

Wilco van den Heuvel and Albert P.M. Wagelmans

ERS-2003-066-LIS

http://hdl.handle.net/1765/930

Arrival Processes for Vessels in a Port Simulation

Eelco van Asperen, Rommert Dekker, Mark Polman, Henk de Swaan Arons \& Ludo Waltman

ERS-2003-067-LIS

http://hdl.handle.net/1765/973

The distribution-free newsboy problem with resalable returns

Julien Mostard, Rene de Koster and Ruud Teunter

ERS-2003-068-LIS

http://hdl.handle.net/1765/975

A note on a multi-period profit maximizing model for retail supply chain management

Wilco van den Heuvel and Albert P.M. Wagelmans

ERS-2003-072-LIS

http://hdl.handle.net/1765/991

The Impact of Complexity, Rate of Change and Information Availability on the Production Planning and Control Structure: Evidence from Medium-Sized Dutch Discrete Manufacturing Firms

Marcel van Assen and Steef van de Velde

ERS-2003-083-LIS

http://hdl.handle.net/1765/1033

The impact of innovation and organizational factors on APS adoption: Evidence from the Dutch discrete parts industry

Bart van Hezewijk, Marcel van Assen and Steef van de Velde

ERS-2003-084-LIS

http://hdl.handle.net/1765/1067 
Equilibrium Constrained Optimization Problems

S. Birbil, G. Bouza , J.B.G. Frenk and G. Still

ERS-2003-085-LIS

http://hdl.handle.net/1765/1068

Network-Based Business Process Management: Embedding Business Logic In Communications Networks L-F Pau and P.H.M.Vervest

ERS-2003-086-LIS

http://hdl.handle.net/1765/1070 